\title{
Review of Ultrasound-Guided Radial Artery Catheter Placement
}

\author{
Andrew G Miller RRT-ACCS and Amy J Bardin MSc RRT
}

\author{
Introduction \\ Radial Artery Cannulation \\ Ultrasound Techniques \\ Discussion \\ Conclusions
}

\begin{abstract}
Radial artery catheters are commonly placed for continuous blood pressure monitoring, frequent arterial blood gas analysis, or frequent blood sampling for diagnostic testing. Radial artery cannulation can be challenging and ultrasound guidance has emerged as a valuable adjunct for the placement of radial artery catheters. The advantages of ultrasound guidance include: real-time visualization of landmarks, improved pre-procedure planning, reduction in complications, less time spent at the bedside, and improved first-attempt success rates. Disadvantages of ultrasound guidance include: equipment cost, equipment availability, limited availability of experts to train providers, and the cost of training providers. Ultrasound machines are readily available and widely utilized in many emergency departments, operating rooms, and ICUs. We will summarize the use of ultrasound guidance for the placement of radial artery catheters and describe the techniques used during ultrasound-guided arterial catheter placement. Training on the use of ultrasound should be encouraged for all practitioners who place radial artery catheters. Key words: radial artery cannulation; ultrasound guidance; arterial catheter placement; ultrasound-guided arterial catheter placement. [Respir Care 2016;61(3):383-388. (C) 2016 Daedalus Enterprises]
\end{abstract}

\section{Introduction}

Radial artery catheters are commonly placed for continuous blood pressure monitoring, frequent arterial blood gas analysis, or frequent blood sampling for diagnostic testing. Current techniques used for radial artery cannulation include: puncturing the vessel and then using the

\footnotetext{
Mr Miller is affiliated with Duke University Medical Center, Durham, North Carolina. Ms Bardin is affiliated with Banner Estrella Medical Center, Phoenix, Arizona.

Ms. Bardin has disclosed a relationship with Telefex. Mr Miller has disclosed no conflicts of interest.

Correspondence: Andrew G Miller RRT-ACCS, Duke University Medical Center, Respiratory Care Services, PO Box 3911, 2301 Erwin Rd, Durham NC 27710. E-mail: Andrew.g.miller@ duke.edu.
}

DOI: $10.4187 /$ respcare 04190
Seldinger technique to advance the catheter over a guidewire, puncturing the vessel and then directly advancing the catheter into the artery, a surgical cutdown to provide direct vessel visualization, use of Doppler ultrasound, and direct vessel visualization with 2-dimensional ultrasound guidance. ${ }^{1,2}$

Ultrasound guidance has emerged as a valuable adjunct for radial artery catheter placement. The advantages of ultrasound guidance include: real-time visualization of landmarks, improved pre-procedure planning, reduction in complications, less time spent at the bedside, and improved first-attempt success rates. Disadvantages of ultrasound guidance include: equipment cost, equipment availability, limited availability of experts to train providers, and the cost of training providers (Table 1). Ultrasound machines are readily available and widely utilized in many emergency departments, operating rooms, and ICUs. We will summarize the available literature describing the use of ultrasound guidance for the placement of radial artery cath- 
Table 1. Advantages and Disadvantages of Ultrasound Guidance

\begin{tabular}{ll}
\hline \hline \multicolumn{1}{c}{ Advantages } & \multicolumn{1}{c}{ Disadvantages } \\
\hline $\begin{array}{l}\text { Real-time visualization of vessel } \\
\text { Improved pre-procedure planning }\end{array}$ & Equipment expense \\
Increased success rates & Equipment availability \\
Reduced complications & Training of practitioners \\
Reduced time spent placing the & Requirement for skilled \\
catheter & practitioners for training
\end{tabular}

eters and describe the techniques used during ultrasoundguided arterial catheter placement.

\section{Radial Artery Cannulation}

According to the Centers for Disease Control and Prevention, the radial artery is the first choice for arterial catheter placement due to its superficial location and the presence of redundant collateral blood supply to the hand via the ulnar artery in most patients. ${ }^{3}$ Adequate collateral circulation from the ulnar artery should be assessed prior to catheter insertion by using the Allen test or modified Allen test. The traditional method for radial artery catheter placement is to locate the vessel via palpation of the pulse or anatomic landmarks. Unfortunately, anatomic landmarks may not locate the radial artery in up to $30 \%$ of patients. ${ }^{4}$ In patients with severe hypotension, morbid obesity, and atherosclerosis, the radial pulse may be weak or absent, making locating the artery via palpation difficult. Other difficulties commonly encountered during radial artery catheter placement include: the inability to thread the wire, hematoma formation, scarring from previous arterial catheters, atherosclerosis, and arterial spasm. There is significant morbidity associated with catheter placement because the procedure often involves a significant amount of pain and anxiety. Other causes of morbidity include: pseudoaneurysm formation, hematoma formation, hand ischemia resulting from vessel damage during insertion attempts due to inadequate collateral circulation from the ulnar artery, thrombus formation, and infection from improper sterile technique.

Eisen et $\mathrm{al}^{5}$ found a success rate of $79 \%$ for radial artery catheter placement when performed by junior medical residents, a $43 \%$ failure rate for female subjects, and a 5.6\% failure rate in male subjects. The authors concluded that low systolic blood pressure and poorly palpable radial pulse were correlated with lower success. ${ }^{5}$ Martin et $\mathrm{al}^{6}$ showed a 98\% (129/132) success rate for radial artery catheter placement; however, their success rate within 3 puncture attempts was 78\% (99/132), and all subjects had a palpable radial pulse. A study by anesthesiologists found a radial artery cannulation success rate of $90 \%$; however, when the pulse was weak, the success rate dropped to $75 \% .^{7}$
The Centers for Disease Control and Prevention currently recommends that central venous catheters be placed via ultrasound whenever the technology is available. ${ }^{3}$ Ultrasound guidance has been shown to reduce complications and increase success rates for bedside clinicians, including respiratory therapists (RTs), during central venous catheter insertion. ${ }^{3-6}$

Ultrasound guidance has been proven to be beneficial for radial artery catheter placement and can be utilized effectively by RTs. ${ }^{1,8-12}$ In the most recent systematic review and meta-analysis, which included 447 ultrasound attempts versus 455 palpation attempts, Gao et $\mathrm{al}^{13}$ concluded that ultrasound significantly increased first-attempt success rate. For all subjects, ultrasound was associated with a risk ratio of 1.47 for first-attempt success; for adult subjects, the risk ratio was 1.38 , and for pediatric subjects, the risk ratio was $1.73 .{ }^{13} \mathrm{In}$ another recent meta-analysis, Tang et al ${ }^{14}$ also assessed first-attempt success rate and found a risk ratio of 1.98 versus 1.38 for experienced versus inexperienced operators. They concluded that ultrasound guidance reduced the mean number of attempts required for successful catheter placement, reduced time to successful catheter placement, and reduced the number of hematomas. Seto et al ${ }^{15}$ randomized 698 subjects to palpation versus ultrasound guidance for radial artery catheterization by experienced interventional cardiologists. They found an increase in first-attempt success rate $(65 \%$ vs $44 \%$ ), a decreased need for 5 or more attempts to gain access (2\% vs 19\%), reduced time to access (88 vs $108 \mathrm{~s}$ ), and fewer attempts (1.7 vs 3.1). In a similar trial enrolling 192 subjects, Zaremski et al ${ }^{16}$ found no difference in success rate ( $87 \%$ vs $87 \%$ ) between ultrasound guidance and palpation methods. It is important to note that all attempts were made by a single experienced interventional cardiologist. Their results indicated a possible benefit to ultrasound guidance for subjects with weak or absent pulses and obese subjects. ${ }^{16}$

Ultrasound guidance may reduce catheter-related infections due to a reduction in attempts and increased firstattempt success rate. ${ }^{13,14}$ Ultrasound guidance reduces the likelihood of hitting the nerve bundle and other structures near the artery due to real-time visualization of the needle as it approaches the artery and underlying structures. This should reduce the morbidity associated with radial artery catheter placement because the procedure can be associated with significant pain, especially when catheter placement requires multiple attempts. To our knowledge, the morbidity associated with insertion has not been specifically evaluated in any studies; however, the meta-analysis from Tang et $\mathrm{al}^{14}$ suggests that there is less morbidity (fewer attempts, hematomas, and less time for successful catheter placement) with insertion when ultrasound guidance is used. 
Although it has not been studied specifically, ultrasound guidance is likely to be helpful in training less experienced providers to place radial artery catheters. The radial artery is small and is difficult to locate in patients with hypotension, shock, atherosclerosis, and obesity. By utilizing ultrasound guidance practitioners can develop necessary skills, such as redirecting of the needle, proper needle depth, and wire placement without the difficulty of puncturing the artery. From our anecdotal experience, it is difficult to teach radial artery catheter placement when the learner is having difficulty puncturing the artery. Ultrasound should minimize this difficulty and reduce the risk of vessel damage and morbidity because real-time guidance reduces the likelihood of hitting underlying structures, such as nerves, ligaments, and tendons. Incorporating ultrasound training into arterial catheter placement training may increase ultrasound utilization and reduce complications during training. This strategy has not been studied and should be a focus of future research.

The training of practitioners to place radial arterial catheters has not been well studied. We suggest that prior to placing radial artery catheters, RTs should be required to complete a didactic training session with a mannequin arm. Before being allowed to place catheters independently, they should be required to successfully place 5 radial artery catheters under supervision. The didactic training should include ultrasound guidance with proficiency in ultrasound guidance demonstrated prior to placing radial artery catheters independently. At Duke University Medical Center, ultrasound guidance is used primarily by RTs in the emergency department, since our emergency department RT core team places approximately 400 radial arterial catheters annually. The decision to utilize ultrasound is left to staff discretion, and we tend to use the technology when difficulty has been encountered or is suspected. Data from our institution found an $86 \%$ success rate and indicated that prior attempts, sex, and systolic blood pressure were not predictors of success. ${ }^{10}$ Staff are strongly encouraged to learn to use ultrasound; however, at this time, ultrasound training is voluntary.

In our institution, RTs certified in radial artery catheter placement complete a bedside training session lasting approximately $30 \mathrm{~min}$ while being proctored by an emergency medicine physician or RT trained in ultrasoundguided arterial catheter placement. The training session includes: ultrasound machine operation, identification of the radial artery, demonstration of the transverse and longitudinal methods, proper sterile technique, and proper left-hand technique to prevent probe movement during attempts. RTs are encouraged to perform arterial puncture with ultrasound guidance and to observe central venous catheter placement or peripheral intravenous placement under ultrasound guidance to improve their understanding of the technique. During their initial attempts, the precep-

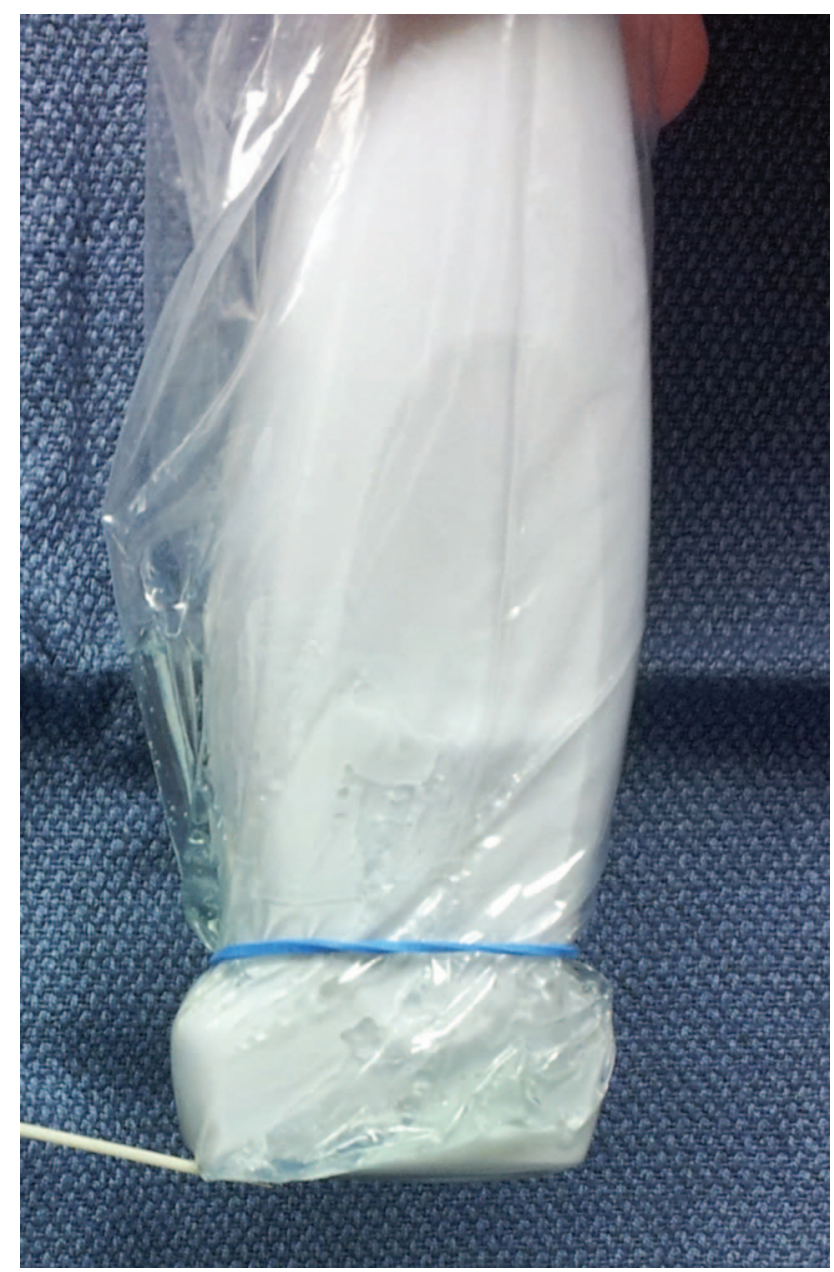

Fig. 1. Example of an ultrasound probe prepped for use.

tor holds the ultrasound probe and guides the therapist's attempt as necessary. Each therapist is expected to demonstrate the ability to utilize ultrasound guidance without an assistant. Each RT is proctored for 3-5 successful catheter placements or until they feel they can perform the procedure without observation. Currently, yearly competence is not being evaluated.

\section{Ultrasound Techniques}

The radial artery is identified using a linear or vascular probe (Fig. 1). The probe is placed on the wrist where the pulse is palpated (or via anatomic landmarks if there is no palpable pulse) or along the forearm and wrist. The artery will be an anechoic or dark spot that can be differentiated from veins due to pulsation of the vessel. Compression of the vessel will make the pulsation of the artery easier to detect, although too much pressure may collapse the vessel in patients with severe hypotension. Poor blood flow may make differentiating artery from vein difficult, and 


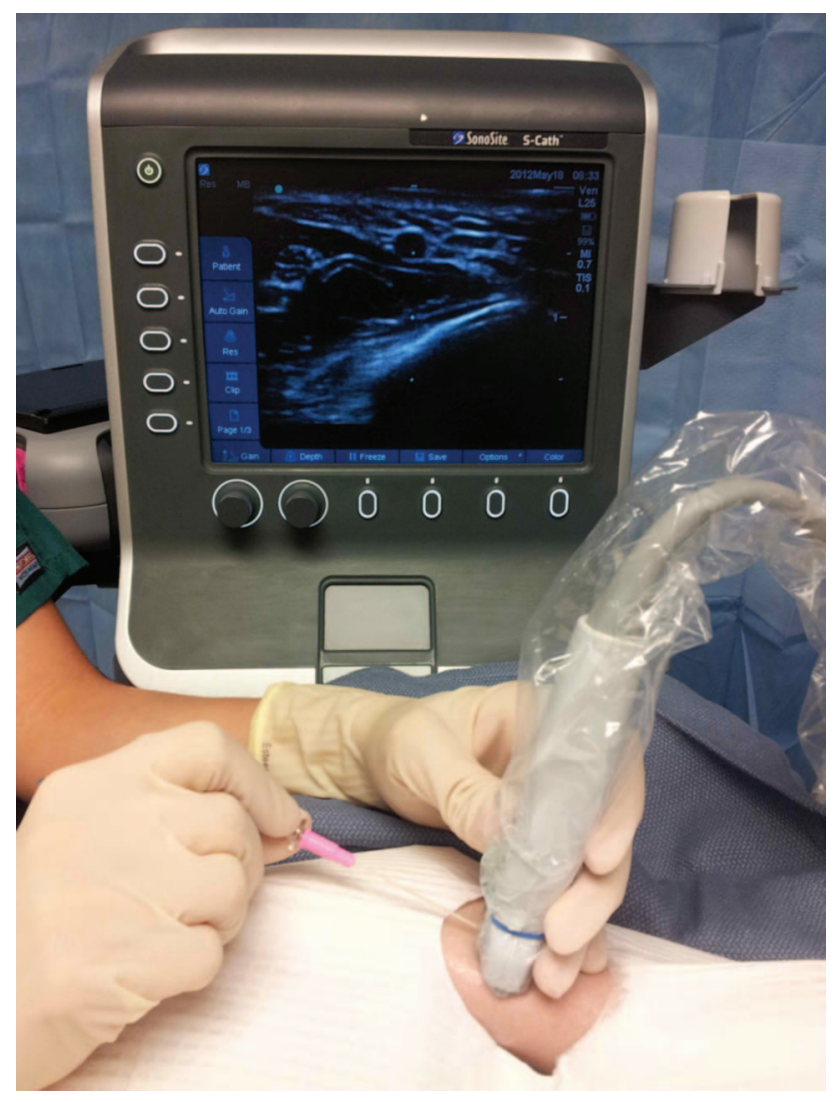

Fig. 2. Transverse view of radial artery. The arrow on the probe corresponds to the guide on the screen of the ultrasound machine; each mark on the guide corresponds to $1 \mathrm{~cm}$ of distance.

many ultrasound machines have a Doppler mode to evaluate blood flow to aid in differentiation. Vessel visualization can be improved by adjusting the depth on the ultrasound machine to the minimum depth and adjusting the gain.

There are 3 primary techniques used for radial artery cannulation. The first is the transverse method, where the probe is placed perpendicular to the artery (Fig. 2). The skin is punctured at a $90^{\circ}$ angle to the point on the probe that corresponds to the guide in the center of the screen. The needle will enter the screen from the center if properly oriented. Special care must be taken to maintain the probe perpendicular to the vessel to ensure proper alignment of the needle and vessel image, or the needle may miss the vessel. By using real-time guidance, the needle can be redirected toward the vessel if the vessel is missed on the first pass.

The second technique is the longitudinal method, where the probe is oriented parallel to the vessel, and the vessel appears as a darkened area on the screen (Fig. 3). The skin is punctured parallel to the probe in the exact center of the probe. The needle will enter the screen from either the left or right, depending on the probe's orientation. The needle

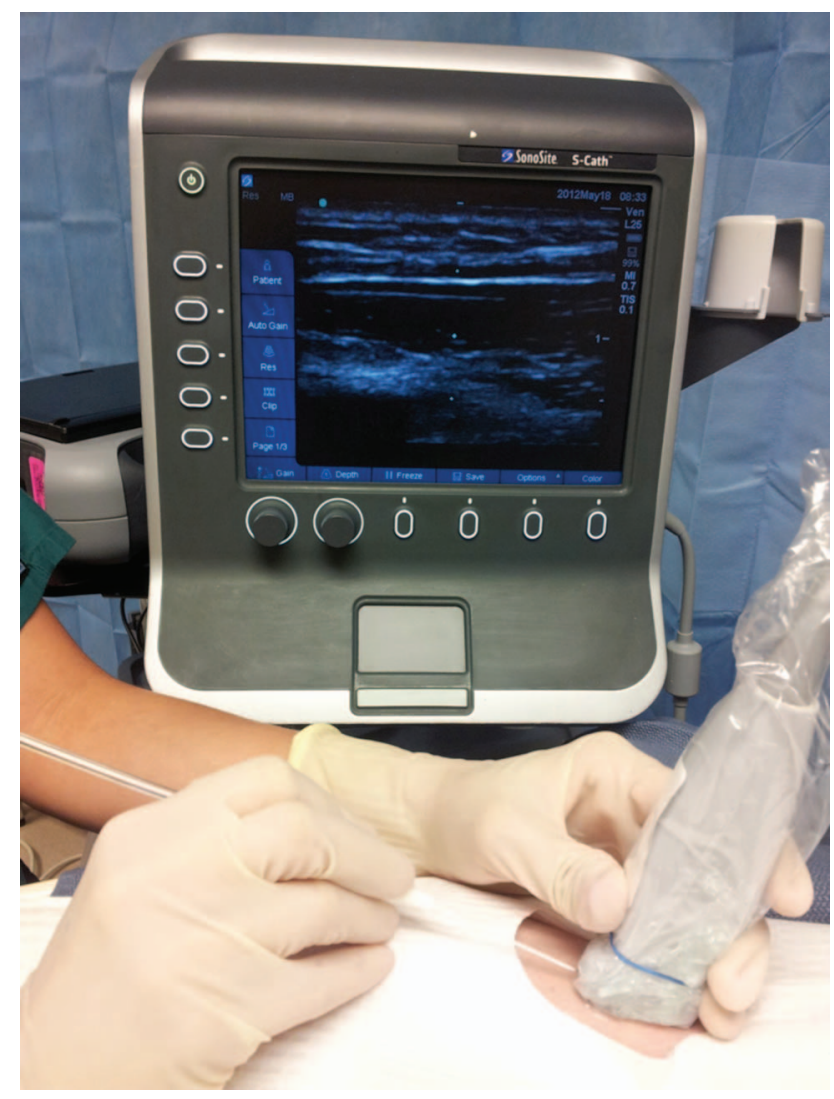

Fig. 3. Longitudinal view of radial artery. The blue dot on the upper left side of the screen corresponds to a mark on the probe. The needle will enter from the left side of the screen.

will be visible entering the vessel when it reaches the correct depth. In some cases, the needle may lie parallel to the artery but may appear to have penetrated it on ultrasound images, in which case no blood return will be seen within the catheter chamber. In this case, the needle should be retracted, and the probe and catheter should be recentered on the long axis of the artery, followed by reinsertion of the catheter.

Berk et $\mathrm{al}^{17}$ evaluated transverse versus longitudinal method in subjects undergoing cardiac surgery. They found that the longitudinal technique reduced time to successful catheter placement ( $24 \mathrm{~s}$ vs $47 \mathrm{~s}$ ), increased first-attempt success rate ( $71 \%$ vs $51 \%$ ), and resulted in fewer complications. Any practitioner who utilizes ultrasound guidance should be able to use both techniques. It has been our experience that the longitudinal method is more difficult to learn and master than the transverse axis method. Once the longitudinal method has been mastered, it may be superior to the transverse method because the needle and catheter can be better visualized entering the vessel. We have used this method to successfully place catheters in patients in whom a flash of blood was never observed in the needle. 
Quan et al ${ }^{18}$ also evaluated transverse versus longitudinal axis in subjects undergoing liver or splenic surgery. One hundred sixty-three subjects were randomized to have radial artery catheters placed either by the transverse or longitudinal method. The first-attempt success rate was higher in the short-axis group ( $89 \%$ vs $73 \%, P=.02$ ), and the failure rate was lower ( $0 \%$ vs $2.4 \%$ ) in the long-axis group. There was no difference in the time required for successful catheter placement or complications between the 2 groups. ${ }^{18}$ It should be noted the mean arterial pressure was 83 and $79 \mathrm{~mm} \mathrm{Hg}$ in the 2 groups. The best method to use is still unclear, and the best strategy may be to utilize whichever technique the operator is most comfortable and experienced utilizing.

A third method is the static technique, where the vessel is identified via ultrasound, and a mark is made on the skin along the artery's path with a sterile marker. This technique may be effective, but it negates the advantage of real-time guidance. This technique has been shown to be less effective than real-time 2-dimensional guidance for internal jugular vein central venous catheters. ${ }^{4}$

Sterile technique should be strictly maintained while placing radial artery catheters because arterial catheters carry a similar risk of catheter-related bloodstream infection as central venous catheters. ${ }^{19,20}$ Programs proven to reduce catheter-related bloodstream infection include a 5-step program with: (1) web-based education on proper insertion methods, (2) placement of all necessary equipment into a catheter-specific cart, (3) use of a daily checklist during rounds concerning the need for continued catheterization, (4) use of a checklist by a nurse during catheter placement to ensure compliance with best practice, and (5) empowerment of nurses to stop the procedure if insertion guidelines or the sterile field is violated. ${ }^{20}$ The use of chlorhexidine-impregnated sponges may reduce catheter-related bloodstream infection. ${ }^{21,22}$ Self-adhesive bandages are available that include a chlorhexidine-impregnated patch. Additional strategies to reduce catheter-related bloodstream infection include: assuring proper hand hygiene, placing a sign on the door indicating that a sterile procedure is in progress, and ensuring that everyone in the room during the procedure wears a cap and mask. Applying the techniques designed to reduce central venous catheter-related bloodstream infection to radial artery catheter placement may reduce catheter-related bloodstream infections, although this has not been directly studied.

For radial artery catheter insertion, the Centers for Disease Control and Prevention recommends barrier precautions that include a hat and mask, skin preparation with $2 \%$ chlorhexidine, and an adequate sterile field over the insertion site. ${ }^{3}$ A sterile probe cover is required when using ultrasound for catheter insertion. An appropriate probe cover encloses the probe itself and the majority of the

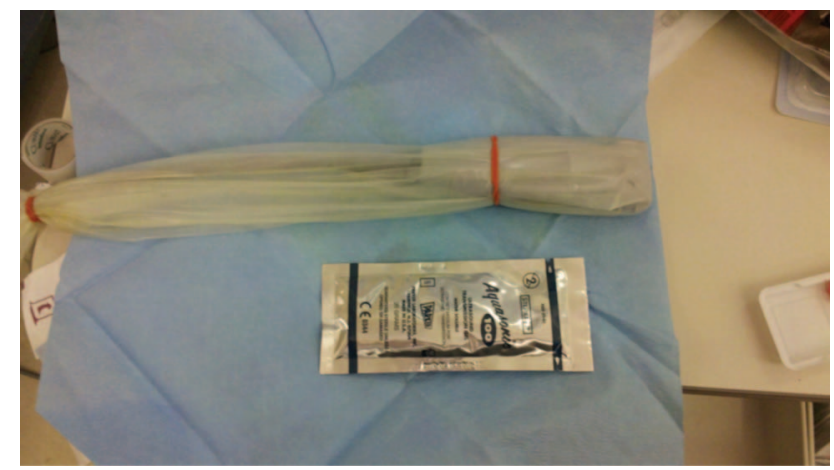

Fig. 4. Example of sterile probe cover prepped for use.

cable, preventing contamination of the sterile field (Fig. 4). The sterile field will maintain insertion site integrity and allow contact of the ultrasound probe without skin contamination. Special care must be taken to avoid contamination of the site selected for cannulation. The ultrasound probe should be held in the non-dominant hand to ensure sterility and practitioner comfort. It may be beneficial to have an assistant hold the ultrasound probe to allow the clinician placing the arterial catheter the use of both hands. This provides the opportunity to visualize the guide wire and catheter entering the vessel, whereas a single practitioner must set the ultrasound probe down in order to thread the wire into the vessel, preventing direct visualization. The assistant must adhere to all guidelines for sterile technique.

\section{Discussion}

In patients who present difficult vascular access, ultrasound guidance is effective for establishing both venous and arterial access. Proper training is essential for successful utilization of ultrasound guidance. The radial artery can be difficult to cannulate, especially for those not experienced in the procedure. ${ }^{6}$ We support the use of ultrasound guidance to reduce insertion-related complications. Ultrasound guidance has become the standard of care for placement of internal jugular vein central venous catheters and peripherally inserted central catheters; current evidence suggests that ultrasound guidance should be the standard of care for radial artery cannulation as well. The technique can easily be used for arterial puncture and may reduce morbidity associated with arterial puncture and assist in success for patients who are considered "tough sticks." Patient populations where ultrasound should be considered are summarized in Table 2.

\section{Conclusions}

Based upon current evidence, ultrasound guidance should be utilized routinely for the placement of radial arterial 


\section{Ultrasound-Guided Radial Artery Catheter Placement}

Table 2. Patient Populations

Patient Populations Where Ultrasound Should Be Considered

Patients without a palpable radial pulse

Patients who have undergone prior attempts

Patients with coagulopathy or abnormal clotting factors

Patients with severe hypotension

Patients with radial artery as the only available site

catheters. Training on the use of ultrasound should be encouraged for all practitioners who place radial artery catheters.

\section{REFERENCES}

1. Levin PD, Sheinin O, Gozal Y. Use of ultrasound guidance in the insertion of radial artery catheters. Crit Care Med 2003;31(2):481484.

2. Brannam L, Blaivas M, Lyon M, Flake M. Emergency nurses' utilization of ultrasound guidance for placement of peripheral intravenous lines in difficult-access patients. Acad Emerg Med 2004;11(12): 1361-1363.

3. O'Grady NP, Alexander M, Burns LA, Dellinger EP, Garland J, Heard SO, et al. Guidelines for prevention of intravascular catheterrelated infections, 2011. Clin Infect Dis 2011;52(9):e162-e193.

4. Brzezinski M, Luisetti T, London MJ. Radial artery cannulation: a comprehensive review of recent anatomic and physiologic investigations. Anesth Analg 2009;109(6):1763-1781.

5. Eisen LA, Minami T, Berger JS, Sekiguchi H, Mayo PH, Narasimhan M. Gender disparity in failure rate for arterial catheter attempts. J Intensive Care Med 2007;22(3):166-172.

6. Martin C, Saux P, Papazian L, Gouin F. Long-term arterial cannulation in ICU patients using the radial artery or dorsalis pedis artery. Chest 2001;119(3):901-906.

7. Karacalar S, Ture H, Baris S, Karakaya D, Sarihasan B. Ulnar artery versus radial artery approach for arterial cannulation: a prospective comparative study. J Clin Anesth 2007;19(3):209-213.

8. Shiloh AL, Savel RH, Paulin LM, Eisen LA. Ultrasound-guided catheterization of the radial artery: a systematic review and metaanalysis of randomized controlled trials. Chest 2011;139(3):524-529.

9. Shiver S, Blaivas M, Lyon M. A prospective comparison of ultrasound-guided and blindly placed radial arterial catheters. Acad Emerg Med 2006;13(12):1275-1279.
10. Miller AG, Cappiello JL, Gentile MA, Almond AM, Thalman JJ, MacIntyre NR. Analysis of radial artery catheter placement by respiratory therapists using ultrasound guidance. Respir Care 2014; 59(12):1813-1816.

11. Sandhu NS, Patel B. Use of ultrasonography as a rescue technique for failed radial artery cannulation. J Clin Anesth 2006;18(2):138141.

12. Schwemmer U, Arzet HA, Trautner H, Rauch S, Roewer N, Greim C. Ultrasound-guided arterial cannulation in infants improves success rate. Eur J Anaesthesiol 2006;23(6):476-480.

13. Gao YB, Yan JH, Gao FQ, Pan L, Wang XZ, Lv CJ. Effects of ultrasound-guided radial artery catheterization: an updated metaanalysis. Am J Emerg Med 2015;33(1):50-55.

14. Tang L, Wang F, Li Y, Zhao L, Xi H, Guo Z, et al. Ultrasound guidance for radial artery catheterization: an updated meta-analysis of randomized controlled trials. PLoS One 2014;9(11):e111527.

15. Seto AH, Roberts JS, Abu-Fadel MS, Czak SJ, Latif F, Jain SP, et al Real-time ultrasound guidance facilitates transradial access: RAUST (Radial Artery access with Ultrasound Trial). JACC Cardiovasc Interv 2015;8(2):283-291.

16. Zaremski L, Quesada R, Kovacs M, Schernthaner M, Uthoff H. Prospective comparison of palpation versus ultrasound-guided radial access for cardiac catheterization. J Invasive Cardiol 2013;25(10): 538-542.

17. Berk D, Gurkan Y, Kus A, Ulugol H, Solak M, Toker K. Ultrasoundguided radial arterial cannulation: long axis/in-plane versus short axis/out-of-plane approaches? J Clin Monit Comput 2013;27(3):319324.

18. Quan Z, Tian M, Chi P, Cao Y, Li X, Peng K. Modified short-axis out-of-plane ultrasound versus conventional long-axis in-plane ultrasound to guide radial artery cannulation: a randomized controlled trial. Anesth Analg 2014;119(1):163-169.

19. Maki DG, Kluger DM, Crnich CJ. The risk of bloodstream infection in adults with different intravascular devices: a systematic review of 200 published prospective studies. Mayo Clin Proc 2006;81(9):11591171.

20. O'Horo JC, Maki DG, Krupp AE, Safdar N. Arterial catheters as a source of bloodstream infection: a systematic review and meta-analysis. Crit Care Med 2014;42(6):1334-1339.

21. Ruschulte H, Franke M, Gastmeier P, Zenz S, Mahr KH, Buchholz $S$, et al. Prevention of central venous catheter related infections with chlorhexidine gluconate impregnated wound dressings: a randomized controlled trial. Ann Hematol 2009;88(3):267-272.

22. Ho KM, Litton E. Use of chlorhexidine-impregnated dressing to prevent vascular and epidural catheter colonization and infection: a meta-analysis. J Antimicrob Chemother 2006;58(2):281-287. 